\title{
Adsorption of Crystal Violet to the Silica-Water Interface Monitored by Evanescent Wave Cavity Ring-Down Spectroscopy
}

\author{
Andrew M. Shaw \\ School of Chemistry, University of Exeter, Stocker Road, Exeter, EX4 4QD, U.K. \\ Theresa E. Hannon, Fuping Li, and Richard N. Zare* \\ Chemistry Department, Stanford University, Stanford, California 94305
}

Received: December 5, 2002; In Final Form: April 16, 2003

\begin{abstract}
Evanescent wave cavity ring-down spectroscopy (EW-CRDS) has been used to investigate the adsorption of crystal violet $\left(\mathrm{CV}^{+}\right)$to a charged silica-water interface as a function of bulk $\mathrm{pH}$ by the direct measurement of the absorbance of the $\mathrm{CV}^{+}$chromophore. Absolute absorbances of order $10^{-4}$ have been routinely detected, showing significant variation in the structure of the silica-water interface. At low ionic strength, the interfacial absorbance of $\mathrm{CV}^{+}$shows a monotonic increase with increasing $\mathrm{pH}$. A simple competitive Langmuir adsorption model, which provides values for the silica surface parameters that are in broad agreement with the existing literature values, has been fit to the data. In addition, interfacial absorbance has been monitored as a function of $\mathrm{pH}$ for $\mathrm{CV}^{+}$solutions maintained at high ionic strength with $\mathrm{NaCl}, \mathrm{KCl}$, and $\mathrm{CaCl}_{2}$. As $\mathrm{pH}$ increases, the $\mathrm{CV}^{+}$interfacial absorbance exhibits a pronounced maximum, which occurs at $\mathrm{pH} 8.7$ for $\mathrm{Na}^{+}$and $\mathrm{K}^{+}$and at pH 7.9 for $\mathrm{Ca}^{2+}$, followed by a sharp decrease. This trend is attributed to competitive binding between the metal cations and $\mathrm{CV}^{+}$to the silica surface binding site, and it has not been observed in previous measurements using second-harmonic generation. The simple Langmuir model, however, does not accurately describe the high ionic strength behavior.
\end{abstract}

\section{Introduction}

Cavity ring-down spectroscopy (CRDS) has proven to be an important technique for the detection of small numbers of molecules in the gas phase ${ }^{1,2}$ by direct absorption. Recently, CRDS has been extended to samples in bulk liquids. ${ }^{3-5}$ It is also possible to use CRDS to detect absorption by species at interfaces often using an attenuated total reflection (ATR) configuration. ${ }^{6-12}$ In particular, Pipino, Hudgens, and Huie ${ }^{8}$ studied iodine at a silica interface using a variation of CRDS called evanescent wave cavity ring-down spectroscopy (EWCRDS). The evanescent wave formed during the total internal reflection (TIR) event may interact with molecules present in the medium of lower refractive index, causing a reduction in the reflected light intensity. CRDS is used to detect a very small change in the absolute absorbance, allowing a significant increase in the sensitivity over that available to conventional ATR experiments. ${ }^{13,14}$ The incorporation of a Dove prism as the TIR element within the ring-down cavity, presented in this paper, allows a particularly simple implementation of EWCRDS.

In this paper, we present an application of the Dove prism cavity to the study of the solid-liquid interface by monitoring the absolute absorbance of a charged chromophore within the silica-water interface structure. The chromophore is attracted to the interface by the surface charge resulting from dissociated surface silanol groups in response to the bulk pH. The structure and absorption spectrum of $\mathrm{CV}^{+}$are shown in Figure 1. The packing of the large chromophore may prevent a simple oneto-one binding on each available site. The absolute absorbance

\footnotetext{
* Corresponding author. E-mail: zare@ @stanford.edu.
}

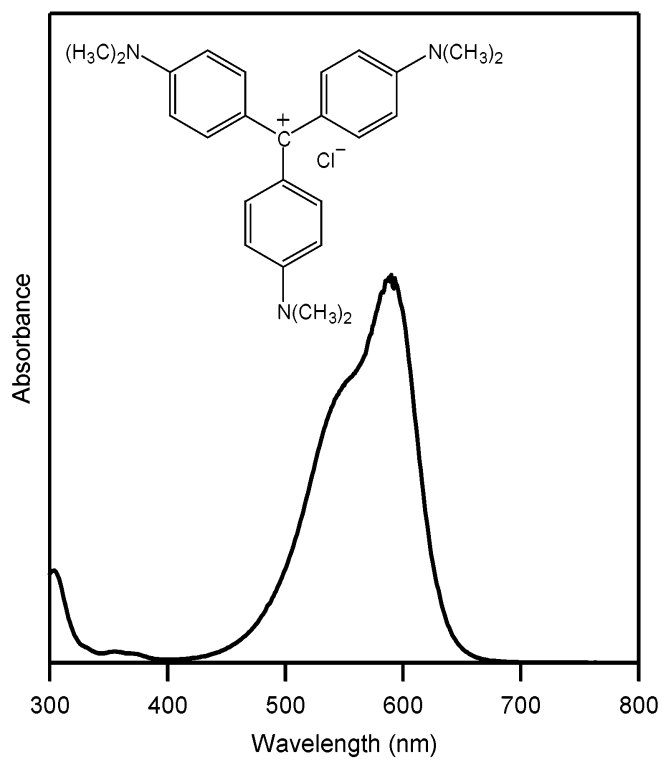

Figure 1. Chemical structure and visible absorption spectrum of $\mathrm{CV}^{+}$ at $\mathrm{pH}>4$.

of the chromophore is monitored as a function of the bulk solution $\mathrm{pH}$ with both low and high ionic strength. The results are interpreted using a Gouy-Chapman mean-field theory of charged electrode interfaces ${ }^{15}$ incorporating a localized binding Langmuir adsorption model for $\mathrm{CV}^{+}$with the silanol sites. The Langmuir model includes simple competitive binding between all positively charged species and the negatively charged dissociated silanol sites. The validity of this model is tested with competitive binding studies that were performed for cations 


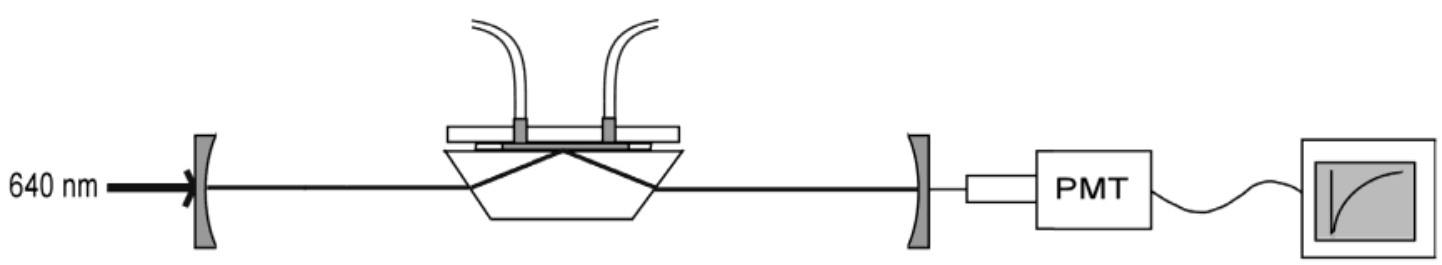

Figure 2. Schematic diagram of the experimental arrangement for Dove prism EW-CRDS.

$\mathrm{Na}^{+}, \mathrm{K}^{+}$, and $\mathrm{Ca}^{2+}$ by adding the chloride salts of these ions to the $\mathrm{CV}^{+}$solutions. The role of surface morphology on the nanometer scale is discussed in the context of the evolution of the interface structure with increasing $\mathrm{pH}$.

\section{Experimental Methods}

EW-CRDS Apparatus. The principles of CRDS have been described elsewhere ${ }^{16}$ and are only briefly mentioned here. Figure 2 shows a diagram of the experimental configuration used in the EW-CRDS measurements. An optical resonator was constructed using two high-reflectivity $(R>99.99 \%$, Los Gatos Research) mirrors placed opposite one another to form a linear cavity. A pulse of radiation entering the cavity through the back of one of the mirrors makes many round-trips, losing some intensity at each bounce. Light leaking from the cavity is detected by a photomultiplier (R4632, Hamamatsu Corporation) and decays exponentially with a $1 / e$ ring-down lifetime, $\tau$, which is of order $15 \mu \mathrm{s}$ for an empty cavity. A Dove prism with antireflection coatings $(R<0.25 \%)$ on the entrance and exit faces (Melles Griot, BK7 glass) was introduced as the TIR element within the cavity. The finesse of the cavity containing the prism is reduced, but ring-down lifetimes of order 150$200 \mathrm{~ns}$ were obtained for a clean prism surface, which allowed a dynamic range of 45-200 ns. The lower end of this range represents a limit determined by the digitization rate and hence the number of points available to determine $\tau$. The maximum $\tau$ corresponds to around 70 passes over the same sample surface area per ring-down lifetime or some 280 passes during the entire ring-down profile.

A flow cell was constructed above the prism surface to allow the prepared solutions to be passed over the TIR interface. The flow cell was made from Plexiglas and sealed to the TIR surface with a silicone gasket. The flow cell had an estimated volume of $100 \mu \mathrm{L}$. The silanol groups on the surface of the gasket were passivated following the procedure of Wadkins and Ligler ${ }^{17}$ using methyltrimethoxysilane to cap the silanol sites on the silicone gasket, preventing unwanted adsorption of $\mathrm{CV}^{+}$during the experiments. The extent of passivation, however, was not determined. Solutions of known $\mathrm{pH}$ were prepared outside the flow cell and pumped over the TIR interface with an HPLC pump (Beckman 110A) connected with Teflon tubing.

Crystal Violet Solutions. Sample solutions were prepared using crystal violet (Aldrich ACS reagent, 95\%) that was stored in a desiccator and protected from light. $\mathrm{NaCl}, \mathrm{KCl}$, and $\mathrm{CaCl}_{2}$ (Aldrich Chemical Co.) were used as received. The solvent for all sample solutions was ultrapure water (with a measured resistivity of $18 \mathrm{M} \Omega \mathrm{cm}$ ) obtained from a Milli-Q Millipore or Barnstead Nanopure purification system. Small amounts of concentrated $\mathrm{NaOH}$ and $\mathrm{HCl}$ solutions were used to vary the $\mathrm{pH}$ without significantly altering the dye concentration. Solution pH was monitored using a Fisher Accumet AB15 pH meter.

EW-CRDS Interfacial Absorbance Measurements. Careful preparation of the prism surface was required to achieve consistent, reproducible results. The TIR surface of the prism was first soaked in methanol for $15 \mathrm{~min}$ followed by $\mathrm{NaOH}$
(>0.6 M) for $10 \mathrm{~min}$ and $50 / 50 \mathrm{H}_{2} \mathrm{SO}_{4} / \mathrm{HNO}_{3}$ (concentrated) for $15 \mathrm{~min}$. The prism was then rinsed thoroughly with ultrapure water and wiped with methanol. ${ }^{18}$ The flow cell was assembled and flushed with methanol.

Radiation from a dye laser (Spectra-Physics, PDL3) at 640 $\mathrm{nm}$ with a pulse duration of approximately $5 \mathrm{~ns}$ was introduced into the cavity. The initial ring-down lifetime, $\tau_{\mathrm{o}}$, was determined with ultrapure water present within the flow cell. The solution of $\mathrm{CV}^{+}$was prepared at the chosen $\mathrm{pH}$ and ionic strength and then pumped into the flow cell, causing the ringdown lifetime to change to a new value, $\tau$. The absolute interfacial absorbance per pass through the sample, $A$ (with the conventional log scale), was calculated using the equation

$$
2.303 A=\frac{\Delta \tau}{\tau \tau_{0}} \frac{t_{\mathrm{r}}}{2}
$$

where $\Delta \tau=\tau_{\mathrm{o}}-\tau$ is the change in the ring-down lifetime without and with the absorber present in the flow cell and $t_{\mathrm{r}}$ is the round-trip time of the light pulse in the cavity. In general, the absorbance $A$ is related to the absorption coefficient $\alpha$ of the sample at the interface through the equation $2.303 A=\alpha d_{\mathrm{e}}$. The parameter $d_{\mathrm{e}}$ is an "effective path length," which replaces the path length $l$ that is conventionally used for bulk UV-vis absorbance measurements. Using the low-absorption approximation $\left(\alpha d_{\mathrm{e}}<0.1\right)$, one finds that the value of $d_{\mathrm{e}}$ depends on the wavelength and polarization of the incident light, the angle of incidence on the interface, and the indices of refraction of the two dielectric media. ${ }^{13}$ For our experiments, these factors did not vary, and $\alpha d_{\mathrm{e}} \ll 0.1$. In this regime, the Beer-Lambert law is obeyed in the same manner as for ATR, and $d_{\mathrm{e}}$ can be considered to be constant.

These interfacial absorption measurements were repeated for several $\mathrm{pH}$ values varying between 2 and 10.5. Experiments were performed with ascending $\mathrm{pH}$, adjusting the $\mathrm{pH}$ of the stock solution with small quantities of concentrated $\mathrm{NaOH}$, and without cleaning the prism surface at each new $\mathrm{pH}$. The upper limit of the $\mathrm{pH}$ was found to be 10.5 , above which the $\mathrm{CV}^{+}$ becomes oxidized and loses its color. ${ }^{19}$

Bulk Solution Absorbance. Absorbance at $640 \mathrm{~nm}$ was monitored from $\mathrm{pH} 2$ to 10.5 for both a bulk aqueous solution of $\mathrm{CV}^{+}$and a bulk $\mathrm{CV}^{+}$solution containing $0.25 \mathrm{M} \mathrm{NaCl}$ (Figure 3). These data were used for comparison with the interfacial absorption data obtained with EW-CRDS. All bulk absorbance measurements were obtained using a UV-vis spectrophotometer (HP 8453).

\section{Results}

Figure 3 shows the absorbance of 640-nm light by $\mathrm{CV}^{+}$ versus $\mathrm{pH}$ for a bulk solution containing $32.7 \mu \mathrm{M} \mathrm{CV}^{+}$. From $\mathrm{pH} 2$ to 4, the absorbance decreases significantly as the conversion from the protonated form to the structure shown in Figure 1 is completed. ${ }^{20}$ A polynomial fit to these data was used to provide a calibration curve for the extinction coefficient as a function of bulk solution $\mathrm{pH}$. With this information, the 


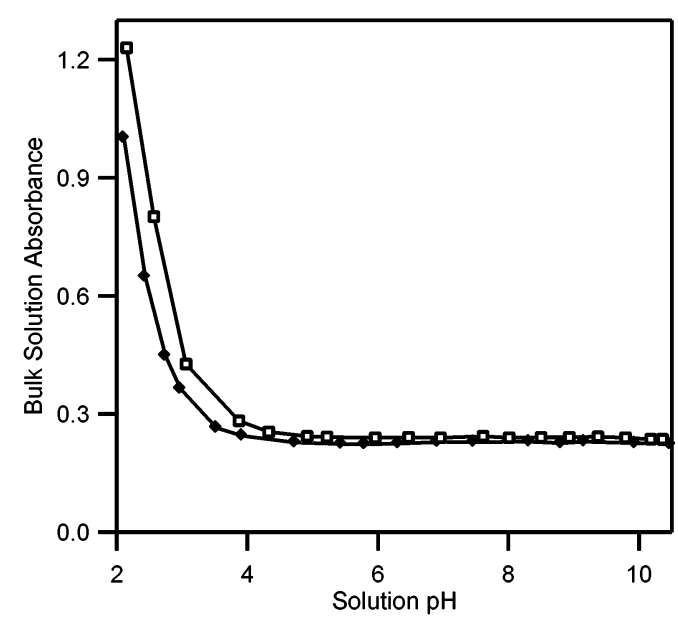

Figure 3. Absorbance at $640 \mathrm{~nm}$ as a function of $\mathrm{pH}$ for $32.7-\mu \mathrm{M}$ crystal violet bulk aqueous solutions $(\square)$ containing no additional salt and $(\diamond)$ containing $0.25 \mathrm{M} \mathrm{NaCl}$.

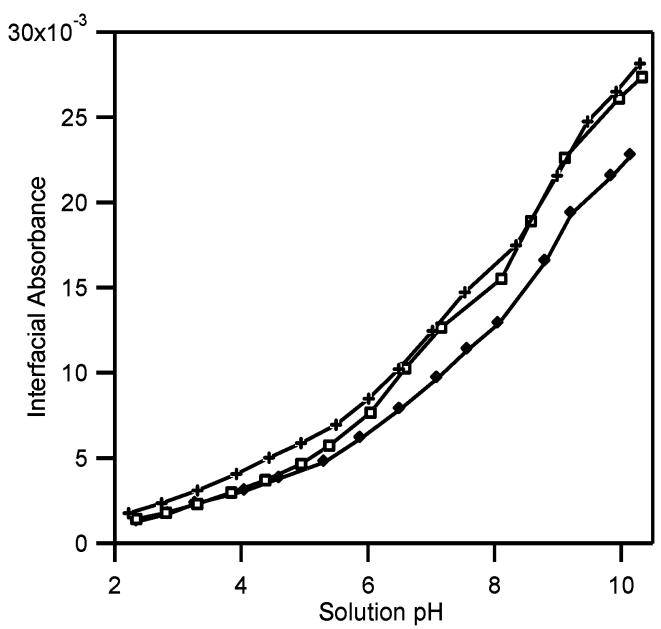

Figure 4. Interfacial absorbance per pass through the sample measured by EW-CRDS plotted as a function of $\mathrm{CV}^{+}$solution $\mathrm{pH}$. Bulk crystal violet solution concentrations are $(\square) 71.3,(\diamond) 69.2$, and $(+) 66.5 \mu \mathrm{M}$. No other salts have been added. (Errors bars of $1 \sigma$ on each point are approximately $1-2 \%$ and are not seen on the graph.)

variation of $\mathrm{CV}^{+}$absorbance as a function of $\mathrm{pH}$ can be eliminated from the interface absorbance measurements. Similarly, the bulk absorbance by $\mathrm{CV}^{+}$in the presence of increased ionic strength $(0.25 \mathrm{M} \mathrm{NaCl})$ is plotted in Figure 3 and shows no significant change with increasing bulk $\mathrm{pH}$. The variation of the bulk extinction coefficient of $\mathrm{CV}^{+}$as a function of bulk $\mathrm{pH}$ may differ from the behavior of the extinction coefficient at the silica-water interface owing to surface $\mathrm{pH}$ values and the presence of the electric field at the charged interface. These possible differences are discussed later.

Figure 4 presents the $640-n m$ EW-CRDS absorbance by $\mathrm{CV}^{+}$ at the silica-water interface as a function of $\mathrm{pH}$. This Figure is in sharp contrast to the trends observed for the changes in bulk absorbance with $\mathrm{pH}$ shown in Figure 3. We present three repeated $\mathrm{CV}^{+}$trials with a cleaned prism surface as outlined above. The error in each of the data points is typically less than $2 \%$, whereas the variation among the three data sets is a measure of the reproducibility of the surface chemistry after the cleaning procedure. The reproducibility is similar for all of the EW-CRDS studies discussed below. The electrolyte concentration increased from the initial $\mathrm{pH} 2$ to the final $\mathrm{pH} 10$ owing to the addition of very small aliquots of $\mathrm{HCl}$ and then $\mathrm{NaOH}$. The overall electrolyte concentration (and hence ionic strength) never

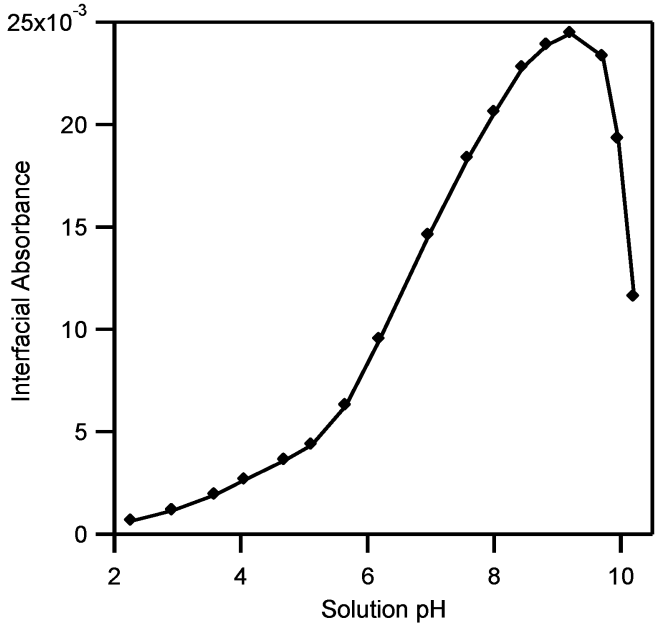

Figure 5. Competition between $\mathrm{CV}^{+}$and $\mathrm{Na}^{+}$at the silica-water interface monitored by EW-CRDS. The bulk solution is $66.5 \mu \mathrm{M}$ crystal violet, $0.25 \mathrm{M} \mathrm{NaCl}$. (Error bars on each point are $\sim 1 \%$.)

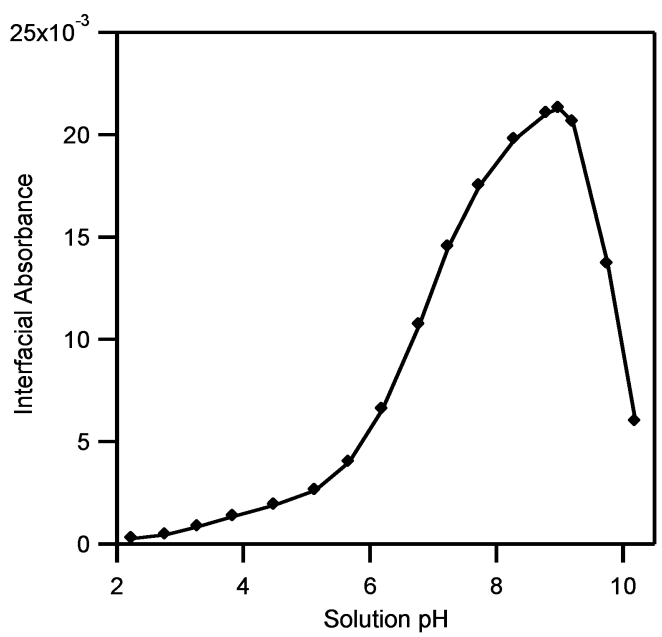

Figure 6. Competition between $\mathrm{CV}^{+}$and $\mathrm{K}^{+}$at the silica-water interface monitored by EW-CRDS. The bulk solution is $66.5 \mu \mathrm{M}$ crystal violet, $0.25 \mathrm{M} \mathrm{KCl}$. (Error bars on each point are $\sim 1 \%$.)

exceeded $30 \mathrm{mM}$ throughout the course of each experiment. For these experiments, the $60-75 \mu \mathrm{M} \mathrm{CV} \mathrm{CV}^{+}$concentration regime was used. In this range, adsorption of $\mathrm{CV}^{+}$to the interface occurred rapidly. After the $\mathrm{CV}^{+}$solutions were flowed over the surface of the prism, the new absorbance value stabilized in less than $30 \mathrm{~s}$.

This 640-nm absorbance by $\mathrm{CV}^{+}$at the silica-water interface shows a monotonically increasing trend with increasing $\mathrm{pH}$. In contrast, Figures 5-7 demonstrate that maintaining a higher bulk ionic strength with a chloride salt produces a maximum in the observed $\mathrm{CV}^{+}$absorbance at alkaline $\mathrm{pH}$. The ionic strength of the solution was kept constant at $0.25 \mathrm{M}$ for the three electrolytes $-\mathrm{NaCl}, \mathrm{KCl}$, and $\mathrm{CaCl}_{2}$. The $\mathrm{CV}^{+}$interfacial absorbance for the $\mathrm{NaCl}$ trial (Figure 5) shows a pronounced maximum at bulk $\mathrm{pH}$ 8.7. The absorbance then decreases rapidly as the $\mathrm{pH}$ is increased. The same trend is observed for the $\mathrm{CV}^{+}$/ $\mathrm{KCl}$ solution (Figure 6), with a maximum $\mathrm{CV}^{+}$absorbance at bulk pH 8.7 followed by a decrease in absorbance to higher $\mathrm{pH}$.

Figure 7 shows interfacial absorbance versus $\mathrm{pH}$ for the $\mathrm{CV}^{+}$ solution containing divalent cation $\mathrm{Ca}^{2+}$ at two different concentrations, 0.083 and $0.25 \mathrm{M}$, that correspond to ionic strengths of 0.25 and $0.75 \mathrm{M}$. The lower-concentration solution, at the same ionic strength as for the $\mathrm{Na}^{+}$and $\mathrm{K}^{+}$experiments, 


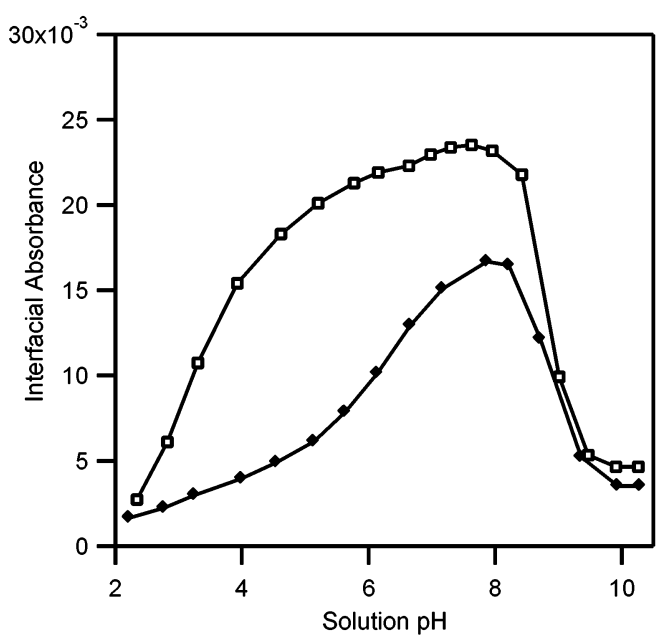

Figure 7. Competition between $\mathrm{CV}^{+}$and $\mathrm{Ca}^{2+}$ at the silica-water interface monitored by EW-CRDS at different salt concentrations. For both curves, the $\mathrm{CV}^{+}$concentration is $66.5 \mu \mathrm{M}$. The $\mathrm{CaCl}_{2}$ concentrations are $(\diamond) 0.083 \mathrm{M}$ (ionic strength $0.25 \mathrm{M}$ ) and $(\square) 0.25 \mathrm{M}$ (ionic strength $0.75 \mathrm{M}$ ). (Errors bars on each point are $\sim 1 \%$.)

shows a trend similar to that observed for the monovalent cations. The absorbance maximum occurs at a somewhat lower $\mathrm{pH}$ value of 7.9. In contrast to Figures 5 and 6 , however, the absolute interfacial absorbance approaches a constant value at a bulk $\mathrm{pH}$ of 10 . The $0.75 \mathrm{M}$ ionic strength solution shows a greater overall absorbance for $\mathrm{CV}^{+}$at the interface with a differently shaped curve. The absorbance rises to a maximum value for lower bulk $\mathrm{pH}$ before showing a pronounced decrease in absorbance at a slightly different $\mathrm{pH}$ value (7.7) followed by leveling out at $\mathrm{pH} 10$.

\section{Discussion}

$\mathrm{CV}^{+}$Adsorption at Low Ionic Strength. We present a model of the silica-water interface that is able to account for the binding of crystal violet as a function of $\mathrm{pH}$, as shown in Figure 4. This model is based on a mean-field theory for describing the potential at an electrode combined with a Langmuir isotherm treatment to describe the variation of the competitive binding of the positively charged chromophore and metal cations with available negatively charged silanol binding sites. This model is able to describe successfully some of our observations but not the behavior of the interface at higher ionic strengths.

The silica-water interface has been studied previously by nonlinear optical techniques, ${ }^{18,21}$ ATR with thermal lens detection, ${ }^{22}$ and fluorescence microscopy. ${ }^{23}$ In addition, the silica surface has been studied by cross-polarization magic angle spinning $\mathrm{NMR}^{24}$ revealing the presence of two different surface ${ }^{29} \mathrm{Si}$ signals having a negative $\delta$ with respect to the solution TMS standard. This observation is consistent with the secondharmonic generation (SHG) studies by Ong, Zhao, and Eisenthal ${ }^{18}$ suggesting that the silica surface contains two types of titratable silanol groups. Group 1 constitutes $19 \%$ of the surface silanol sites and has a $\mathrm{p} K_{\mathrm{a}}(1)$ value of 4.5 ; group 2 comprises the remaining $81 \%$ of the surface sites and has a $\mathrm{p} K_{\mathrm{a}}(2)$ value of 8.5. A study by Dong, Pappu, and $\mathrm{Xu}^{21}$ reported a silanol group density of $8.2 \times 10^{13} \mathrm{~cm}^{-2}$ or 0.8 groups $\mathrm{nm}^{-2}$ (Table 1 ), although estimates ${ }^{25}$ have varied up to 8 groups $\mathrm{nm}^{-2}$. As the bulk $\mathrm{pH}$ is increased, the silica surface becomes more negatively charged, reaching a surface potential with respect to zero in the bulk of $-140 \mathrm{mV}$ at $\mathrm{pH} 10 .{ }^{18}$ The precise estimate of the potential depends, however, on the theoretical model of the
TABLE 1: Parameters for the Silica-Water Interface Obtained from the Langmuir Adsorption Model and Compared with Other Literature Values

\begin{tabular}{lccc}
\hline parameter & $\mu \pm \sigma$ & $\begin{array}{c}\text { previous } \\
\text { work }\end{array}$ & reference \\
\hline$\rho / \mathrm{nm}^{-2}$ & $0.23 \pm 0.01$ & 0.8 & 21 \\
$r$ & $0.39 \pm 0.02$ & 0.19 & 18 \\
$\mathrm{p} K_{\mathrm{a}}(1)$ & $2.1 \pm 0.9$ & 4.5 & 18 \\
$\mathrm{p} K_{\mathrm{a}}(2)$ & $7.50 \pm 0.01$ & 8.5 & 18 \\
$\mathrm{p} K_{\mathrm{CV}^{+}}$ & $-2.16 \pm 0.02$ & -5.2 & 21 \\
$\mathrm{p} K_{\mathrm{Na}^{+}}$ & $-0.60 \pm 0.04$ & -5.2 & 27
\end{tabular}

interface structure. The relationship between charge on an electrode surface and surface potential is described by meanfield theories that are the foundation of the Gouy-Chapman and Stern models of diffuse layers and bilayers. ${ }^{15}$ Ong, Zhao, and Eisenthal ${ }^{18}$ reported the formation of an ionic layer next to the charged surface, and they suggested that the water molecules within the layer are polarized by the electric field of the interface.

Localized binding of a charged chromophore to a binding site at the interface, however, cannot be described simply by a mean-field description. It is conventionally represented by an adsorption isotherm, the simplest of which is the Langmuir isotherm for the description of monolayer adsorption. The size and binding orientation of the chromophore become important in relation to the density of the deprotonated silanol groups. $\mathrm{CV}^{+}$(Figure 1) is a large cation with a surface area of $\sim 120$ $\AA^{2}$, and $\mathrm{CV}^{+}$is believed to lie nearly flat on the silica surface during adsorption. ${ }^{21}$ However, this orientation may be prevented by steric interactions if two silanol sites are close to one another, inhibiting the ion from binding with a flat geometry to both sites. The surface morphology on the nanometer scale may further complicate the interface structure, as has been demonstrated using fluorescence microscopy. ${ }^{23}$ A cationic fluorophore shows a marked propensity for binding at nanometer-scale topographical features on the surface.

The prism surface is polished to $\lambda / 10$ at $633 \mathrm{~nm}$, so we would expect surface morphology on the 60-nm length scale. The penetration depth of the evanescent wave into the solution is approximately $125 \mathrm{~nm}$ in the current configuration. Formally, molecules within the entire electric field of the evanescent wave, which decays exponentially with distance from the surface, will contribute to the measured absorbance. The total EW-CRDS absorbance is the Laplace transform of the concentration profile, $c(z)$, for each angle of incidence. ${ }^{13}$ Calculations using a GouyChapman exponential decay of the potential into the solution suggest that the contribution to the absorbance from molecules further from the surface is smaller, although as the ionic strength increases a bilayer structure will form. A complete description of the chromophore binding must include all of these bindinggeometry factors as the interface structure evolves with increasing surface charge.

The theoretical model used here for the silica-water interface is a simple diprotic surface with two types of silanol group and a group density per unit area. The parameters for the model are based on the SHG studies of a fused silica-water interface by Ong, Zhao, and Eisenthal ${ }^{18}$ and are listed in Table 1 . The density of the silanol groups on the surface, $\rho$, may be related to the surface charge, $\sigma$, by the expression

$$
\sigma=\rho e\left(\frac{r}{1+\frac{\left[\mathrm{H}^{+}\right]_{\mathrm{S}}}{K_{\mathrm{a}}(1)}}+\frac{(1-r)}{1+\frac{\left[\mathrm{H}^{+}\right]_{\mathrm{S}}}{K_{\mathrm{a}}(2)}}\right)
$$


where $r$ is the proportion of group 1 with the lower $\mathrm{p} K_{\mathrm{a}}$ value, $\left[\mathrm{H}^{+}\right]_{\mathrm{s}}$ is the surface concentration of $\mathrm{H}^{+}$, and $K_{\mathrm{a}}(1)$ and $K_{\mathrm{a}}(2)$ are the binding constants for the silanol groups given by

$$
K_{\mathrm{a}}=\frac{\left[\mathrm{SiO}^{-}\right]\left[\mathrm{H}^{+}\right]_{\mathrm{S}}}{[\mathrm{SiOH}]}
$$

The potential at the negatively charged silica-water interface attracts the positively charged chromophore ions, or counterions, enhancing the concentration of these ions at the interface compared with that in the bulk solution. The negatively charged ions, or co-ions, are conversely depleted in the interface compared with their bulk concentrations. The concentration of counterions or co-ions at the surface is given by the Boltzmann distribution ${ }^{15}$

$$
n_{z=0}=n_{\infty} \exp \left(\frac{-q e \phi_{0}}{k T}\right)
$$

where $\phi_{0}$ is the surface potential, $q$ is the charge on the ion (positive or negative), $k$ is the Boltzmann constant, $T$ is the temperature, and $n_{\infty}$ is the concentration of the ionic species in the bulk solution. The Boltzmann factor increases the concentrations of counterions (e.g., $\mathrm{Na}^{+}$) and all positive ions including the protons at the surface by up to 6 orders of magnitude over their bulk concentrations for a surface potential of $-120 \mathrm{mV} .{ }^{26}$ Hence, the surface $\mathrm{pH}$ depends on the surface potential and the surface charge density. Equation 4 also describes the corresponding decrease in the co-ion (e.g., $\mathrm{Cl}^{-}$) concentrations. The co-ions and the counterions balance the surface charge $\sigma$, as expressed by the Grahame equation ${ }^{15}$

$$
\sigma^{2}=2 \epsilon_{\mathrm{r}} \epsilon_{0} k T\left(\sum_{i} n_{i \infty} \exp \left(\frac{-q e \phi_{0}}{k T}\right)-\sum_{i} n_{i \infty}\right)
$$

where $\epsilon_{0}$ is the vacuum permittivity, $\epsilon_{\mathrm{r}}$ is the relative permittivity of the medium, and $n_{i \infty}$ is the bulk concentration of each ionic species, $n_{i}$. For 1:1 electrolytes, this equation reduces to the conventional Gouy-Chapman expression for the potential. ${ }^{15}$ Equations 2 and 5 are combined to determine the potential at the silica surface, $\phi_{0}$.

Increasing the solution $\mathrm{pH}$, therefore, increases the number of dissociated silanol groups, producing an increased surface charge and hence surface potential. The larger number of $\mathrm{SiO}^{-}$ groups provides a population of possible binding sites for the charged chromophore, $\mathrm{CV}^{+}$. Thus, the density of silanol binding sites is a function of $\mathrm{pH}, \rho(\mathrm{pH})$. For the experiment shown in Figure $4, \mathrm{CV}^{+}$is assumed to bind competitively with the $\mathrm{Na}^{+}$ introduced as $\mathrm{NaOH}$ during the preparation of the solutions. The simplest description of the competitive binding is the formation of a monolayer of counterions occupying the available charged silanol sites. This description is given by the Langmuir adsorption model, modified for competitive binding:

$$
\theta=\frac{K_{\mathrm{CV}^{+}}\left[\mathrm{CV}^{+}\right]_{\mathrm{S}}}{1+K_{\mathrm{CV}^{+}}\left[\mathrm{CV}^{+}\right]_{\mathrm{S}}+K_{\mathrm{Na}^{+}}\left[\mathrm{Na}^{+}\right]_{\mathrm{S}}}
$$

$K_{\mathrm{CV}^{+}}$and $K_{\mathrm{Na}^{+}}$are the binding equilibrium constants for $\mathrm{CV}^{+}$ and $\mathrm{Na}^{+}$, and $\theta$ is the fractional surface coverage.

In the case of the data shown in Figure 4, the monolayer adsorption by $\mathrm{CV}^{+}$dominates the observed absorbance. The

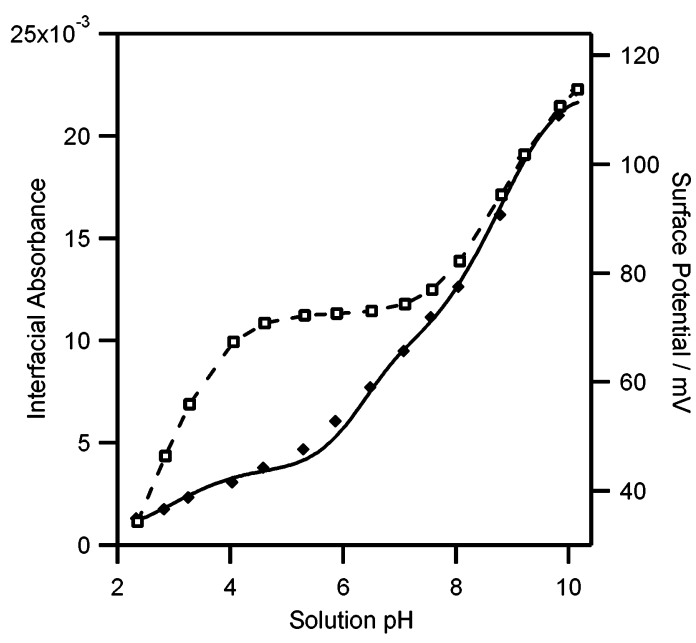

Figure 8. Graph of the Langmuir adsorption model showing $(\bullet)$ the $\mathrm{CV}^{+}$interfacial absorbance data (left axis) that is also plotted in Figure $4,(-)$ the fit of the model to the data, and (- $-\square--)$ the interfacial potentials (right axis) calculated for the different $\mathrm{pH}$ values using the parameters determined from the model.

absorbance of the molecules within a single monolayer is expressed as

$$
A=\rho \theta \sigma_{\mathrm{t}}
$$

where $\rho$ is the surface density of silanol groups, $\theta$ is the fractional surface coverage, and $\sigma_{\mathrm{t}}$ is the absorption cross section of the electronic transition. The cross section is a function of the surface $\mathrm{pH}$ and is related to the molar extinction coefficient $\epsilon(\mathrm{pH})$ (determined from Figure 3) by $\epsilon(\mathrm{pH}) \times 1000 / N_{\mathrm{A}}$ in units of $\mathrm{cm}^{2}$. By replacing $\left[\mathrm{Na}^{+}\right]_{\mathrm{s}},\left[\mathrm{CV}^{+}\right]_{\mathrm{s}}$, and $\left[\mathrm{H}^{+}\right]_{\mathrm{s}}$ with the appropriate Boltzmann distributions and combining eqs 2, 5, 6, and 7, the interfacial absorbance $A$ was modeled as a function of parameters $\rho, r, K_{\mathrm{CV}^{+}}, K_{\mathrm{Na}^{+}}+\mathrm{p} K_{\mathrm{a}}(1)$, and $\mathrm{p} K_{\mathrm{a}}(2)$. The model was fitted to one of the low ionic strength data sets shown in Figure 4, allowing these parameters to vary using a LevenbergMarquardt nonlinear fitting routine. Table 1 presents the results, and Figure 8 shows the fit to the data. Using the values for these parameters, the surface potential $\phi_{0}$ was calculated for each $\mathrm{pH}$ value, as illustrated in Figure 8 .

This Langmuir competitive binding model shows a trend in surface potential with $\mathrm{pH}$ similar to that measured in the SHG experiments of Ong, Zhao, and Eisenthal. ${ }^{18}$ The calculated value of $\phi_{0}$ reaches a maximum near $-120 \mathrm{mV}$ with respect to the bulk liquid potential (assumed to be zero). In addition, the EWCRDS measurements are sensitive to the surface coverage of the $\mathrm{CV}^{+}$chromophore. From the fit to the data, this surface coverage $\theta$ reaches $25 \%$ for the highest potential.

The parameters in Table 1 differ somewhat from those obtained by other methods, ${ }^{18,21,27}$ owing to the experimental conditions of this study and to the assumptions implicit in this model. Although the model assumes that the surface is fused silica, the prism material is BK7 glass and will contain a surface site density and $\mathrm{p} K_{\mathrm{a}}$ values for borate groups. In principle, borate possesses three types of titratable $\mathrm{OH}$ groups, producing a range of site $\mathrm{p} K_{\mathrm{a}}$ values. Variation in the $\mathrm{p} K_{\mathrm{a}}$ values may also be caused by the surface morphology, especially the local extreme surface curvature. Silanol sites in regions of high surface curvature may experience higher electric fields and therefore different surface-group acidities.

The binding coefficients $K_{\mathrm{CV}^{+}}$and $K_{\mathrm{Na}^{+}}$discussed in this paper are different from those determined previously. We observe differences of approximately 3 orders of magnitude 
(Table 1) in the values of $K_{\mathrm{CV}^{+}}$and $10^{4}$ for $K_{\mathrm{Na}}{ }^{+}$compared with the values in other studies. In this study, $K_{\mathrm{CV}^{+}}$and $K_{\mathrm{Na}^{+}}$are defined in terms of concentrations of the ions at the interface, in contrast to previous definitions ${ }^{21,27}$ employing the bulk concentrations of the species, which are significantly smaller than the interfacial values. The Boltzmann factor in eq 4 causes the surface concentrations to be functions of the surface potential and causes a variation in the determined binding coefficients. Further differences may result from comparison with the measurements made in acetonitrile, ${ }^{21,27}$ which will affect the relative permittivity within the bulk and at the interface. The role of the relative permittivity in the model is discussed below. In addition, the surface roughness of the prism and the presence of some borate groups change the values of the measured binding constants as well as the surface availability and density of silanol binding sites.

The model also has some implicit assumptions regarding the electrostatic structure of the interface. This model assumes that the absorbance is dominated by the Langmuir competitive binding of the $\mathrm{CV}^{+}$at the immediate silica surface. Therefore, it ignores the smaller absorbance contributions from molecules that are present in a diffuse layer that evolves to a bilayer as the surface charge increases. The relative permittivity $\epsilon_{\mathrm{r}}$ of the evolving layer describes how the electric field varies throughout the interface, and this quantity is assumed to be constant at the bulk value, $\epsilon_{\mathrm{r}}=78.3$, in this model. The electric field at the interface is rather large; $100 \mathrm{mV}$ dropped over approximately $10 \AA$ is a field of $10^{7} \mathrm{~V} \mathrm{~cm}^{-1}$, which should be sufficient to induce a Stark shift in the absorption spectrum of $\mathrm{CV}^{+}$. In addition, the interfacial absorption spectrum of $\mathrm{CV}^{+}$may be altered by dimerization or intermolecular interactions that result from the increased concentration of the chromophore at the surface.

$\mathbf{C V}^{+}$Adsorption at Higher Ionic Strength. The GouyChapman potential model, modified for Langmuir adsorption as described above, provides an appropriate fit to the low ionic strength interfacial absorbance data, but this model does not reproduce the data shown in Figures 5-7. A better understanding of the evolution of the interface structure as a function of surface charge (and hence $\mathrm{pH}$ ) is required to interpret these competitive binding experiments quantitatively.

The relative sizes of the competing cations become more important in the presence of a higher concentration of ions. The $\mathrm{Na}^{+}, \mathrm{K}^{+}$, and $\mathrm{Ca}^{2+}$ cations are not sterically hindered from binding to all of the available silanol sites with unit occupancy, whereas the $120-\AA^{2} \mathrm{CV}^{+}$ions experience significant steric hindrance. ${ }^{26}$ The different curve shape seen in Figures $5-7$ for the $0.25 \mathrm{M}$ ionic strength studies may be indicative of a change to this Stern layer model. The dramatic turnover point in interfacial absorbance for each of these trials indicates the $\mathrm{pH}$ value at which the $\mathrm{Na}^{+}, \mathrm{K}^{+}$, or $\mathrm{Ca}^{2+}$ cation begins to dominate the competition for anionic surface silanol sites. The location of the absorbance maximum corresponds roughly with the $\mathrm{p} K_{\mathrm{a}}$ of the more prevalent silanol group on the silica surface. $\mathrm{Ca}^{2+}$ dominates the competition with $\mathrm{CV}^{+}$at a lower $\mathrm{pH}$ (7.9) in comparison with either $\mathrm{Na}^{+}$or $\mathrm{K}^{+}$, owing to the higher chargeto-volume ratio of $\mathrm{Ca}^{2+}$ and hence its stronger propensity for binding.

What is less clear, however, is why an enhanced absorbance for $\mathrm{CV}^{+}$at lower $\mathrm{pH}$ occurs in the presence of a much higher $\mathrm{Ca}^{2+}$ concentration (ionic strength $0.75 \mathrm{M}$, shown in Figure 7). Possible future competitive adsorption experiments might involve differently sized chromophores and cations. These studies should allow the roles of competitive binding and ionic strength in the transition from a Gouy-Chapman potential distribution to a Stern layer interface structure to be investigated using EW-CRDS.

\section{Conclusions}

These studies of the silica-water interface using EW-CRDS have provided new experimental insight into the fundamental structure of the charged interface. Competitive binding has been observed between the large surface area chromophore $\mathrm{CV}^{+}$and the much smaller metal cations. This behavior suggests a number of further studies to investigate the binding of differently sized chromophores and competing cations. The stability of a bilayer at the interface in bulk solutions with high ionic strength may be maintained with other bulky cations, producing a simpler capacitor bilayer structure that is more mathematically tractable. Furthermore, the prospect of kinetic measurement with lower concentrations of chromophore would test the Langmuir adsorption isotherm assumptions and should reveal a variation of binding kinetics with bulk $\mathrm{pH}$.

Acknowledgment. We thank Jim Mikkelsen for his help in constructing the flow cell. This work was funded by the Office of Naval Research (grant number N000140010364), and T.E.H. acknowledges support from an Evelyn Laing McBain Fellowship.

\section{References and Notes}

(1) Berden, G.; Peeters, R.; Meijer, G. Int. Rev. Phys. Chem. 2000, 19,565 .

(2) Spence, T. G.; Harb, C. C.; Paldus, B. A.; Zare, R. N.; Willke, B.; Byer, R. L. Rev. Sci. Instrum. 2000, 71, 347.

(3) Hallock, A. J.; Berman, E. S. F.; Zare, R. N. Anal. Chem. 2002, 74,1741 .

(4) Xu, S.; Sha, G.; Xie, J. Rev. Sci. Instrum. 2002, 73, 255.

(5) Snyder, K. L.; Zare, R. N. Anal. Chem. In press, 2003.

(6) Curran, R. M.; Crook, T. M.; Zook, J. D. Mater. Res. Soc. Symp. Proc. 1988, 105, 175 .

(7) Engeln, R.; Berden, G.; van den Berg, E.; Meijer, G. J. Chem. Phys. 1997, 107, 4458.

(8) Pipino, A. C. R.; Hudgens, J. W.; Huie, R. E. Chem. Phys. Lett. 1997, 280, 104

(9) von Lerber, T.; Sigrist, M. W. Appl. Opt. 2002, 41, 3567.

(10) Kleine, D.; Lauterbach, J.; Kleinermanns, K.; Hering, P. Appl. Phys. $B$ 2001, 72, 249.

(11) Gupta, M.; Jiao, H.; O'Keefe, A. Opt. Lett. 2002, 27, 1878.

(12) Muir, R. N.; Alexander, A. J. Phys. Chem. Chem. Phys. 2003, 5, 1279 .

(13) Harrick, N. J. Internal Reflection Spectroscopy; Wiley \& Sons: New York, 1967.

(14) Internal Reflection Spectroscopy: Theory and Applications; Mirabella, F. M., Ed.; Practical Spectroscopy Series; Marcel Dekker: New York, 1993; Vol. 15.

(15) Bard, A. J.; Faulkner, L. R. Electrochemical Methods: Fundamentals and Applications, 2nd ed.; Wiley \& Sons: New York, 2001.

(16) Zalicki, P.; Zare, R. N. J. Chem. Phys. 1995, 102, 2708.

(17) Wadkins, R. M.; Ligler, F. S. Immunosensors Based on Evanescent Wave Excitation. In Affinity Biosensors: Techniques and Protocols; Rogers, K. R., Mulchandani, A., Eds.; Methods in Biotechnology; Humana Press: Totowa, NJ, 1993; Vol. 7, pp 77-87.

(18) Ong, S.; Zhao, X.; Eisenthal, K. B. Chem. Phys. Lett. 1992, 191 327.

(19) Hoener, K. Math. Naturwiss. Unterr. 1998, 51, 286

(20) Green, F. J. The Sigma-Aldrich Handbook of Stains, Dyes, and Indicators; Aldrich Chemical Co.: Milwaukee, WI, 1990; pp 239-240.

(21) Dong, Y.; Pappu, S. V.; Xu, Z. Anal. Chem. 1998, 70, 4730.

(22) Shimosaka, T.; Sugii, T.; Hobo, T.; Ross, J. B. A.; Uchiyama, K. Anal. Chem. 2000, 72, 3532

(23) Wirth, M. J.; Ludes, M. D.; Swinton, D. J. Anal. Chem. 1999, 71, 3911.

(24) Maciel, C. E. In The Encyclopedia of Nuclear Magnetic Resonance; Grant, D. M., Harris, R. K., Eds.; Wiley: Chichester, U.K., 1996; p 4370 (25) Cox, G. B. J. Chromatogr., A 1993, 656, 353.

(26) Intermolecular and Surface Forces, 2nd ed.; Israelachvili, J., Ed.; Academic Press: London, 1992.

(27) Dong, Y.; Xu, Z. Langmuir 1999, 15, 4590. 